\title{
Acute Bone Remodeling after Reduction of Nasal Bone Fracture on Computed Tomography Imaging
}

Bong Moo Lee, Dong Gil Han

Department of Plastic and Reconstructive Surgery, Catholic University of Daegu School of Medicine, Daegu, Korea
No potential conflict of interest relevant to this article was reported.

\begin{abstract}
Background: A number of studies have reported complication after reduction of nasal bone fractures. Among complicated cases, some showed improvement in shape of the nose with passage of time. Therefore, we examined these changes using computed tomography (CT) images taken over intervals.

Methods: CT scans of 50 patients with new nasal bone fractures were reviewed, and the images were compared amongst preoperative, immediately postoperative, and one month scans. Changes in nasal bone shape, were evaluated based on the angle of nasal bone arch between the nasal bone and frontal process of maxilla, overall shape of arch, mal-alignment of fracture segments involving bony irregularity or bony displacement. These evaluations were used to separate postoperative outcomes into 5 groups: excellent, good, fair, poor, and very poor.

Results: Immediate postoperative nasal shape was excellent in 10 cases, good in 31 cases, fair in 8 cases, and poor results in a single case. Postoperative shape at one month was excellent in 37 cases, good in 12 cases, fair in a single case.

Conclusion: The overall shape of nasal bone after fracture reduction tended to improve with passage of time.
\end{abstract}

Keywords: Nasal bone / Surgery / Bone remodeling

\section{INTRODUCTION}

The nasal bone is a prominent feature of the central face and is the most common fracture site in facial bone. Nasal bone fractures account for $40 \%$ of facial bone fractures and is the third most common of all skeletal fractures [1]. If reduction is not performed properly, contracture and fibrosis of soft tissue can result in nasal deformity and functional problems, such as septal deviation, nasal obstruction, and temporary hyposmia [2]. Therefore, exact diagnosis and treatment is important to obtain good outcome. Because nasal bone fracture is considered a minor injury, accurate diagnosis and precise reduction tend to be overlooked, and often

Correspondence: Dong Gil Han

Department of Plastic and Reconstructive Surgery, Catholic University of Daegu School of Medicine, 33 Duryugongwon-ro 17-gil, Nam-gu, Daegu, 705-718, Korea E-mail: dghan1001@cu.ac.kr

Received June 24, 2014 / Revised July 26, 2014 / Accepted August 5, 2014 results in multiple forms of nasal deformities even after the operation [3]. However, we found that nasal deformity from the immediate postoperative period tended to improve with time.

This study analyzes acute bone remodeling or realignment of nasal bone after closed reduction on computed tomography (CT) images taken preoperatively, immediately after the operation, and at one month after the operation. The degree of nasal bone repositioning was analyzed according to fracture types.

\section{METHODS}

\section{Patients}

This study reviewed a period between September 2012 and January 2014. Among 243 patients with pure nasal bone fracture, 50 patients who wished for and underwent repeat CT scans at 1 
month were included in the study.

\section{Surgical technique}

All of the fractures were reduced in a closed manner, within 2 weeks of injury, by the same surgeon under general anesthesia. Following the reduction, vaseline roll gauze was packed in the dorsal nasal cavity to support the reduced bone fragments. Merocels were packed in nasal airways, and external nasal thermo-splint were applied. Packed Merocels were removed six hours after operation, and packed vaseline roll gauzes were removed one day after operation for patient comfort, as we have detailed in a previous study on the effect of early removal of nasal packing in a previous report, which showed that there was no difference in outcomes [3].

\section{Assessment methods}

In the current study, a dual 128-channel CT, SOMATOM Definition flash (Siemens Industry, Munich, Germany) was used. The axial CT images had slice thicknesses of $1 \mathrm{~mm}$ and had been scanned along the nasal bone to the nasal apex. For each patient, we tried to use the same image section of CT scans across the intervals (before, immediately after, and 1 month after operation) to allow as an object comparison of outcomes as possible.

Postoperative outcomes were determined using the following factors: degree of deviation $(\theta$, the angle between the nasal bone and the frontal process of the maxillary bone) (Fig. 1) [4], overall shape of arch, malalignment of fracture segment, bony irregularity, and bony displacement. Using these factors, the outcomes were classified in the manner listed below:

Excellent: deviation of the nasal bone is absent; overall shape of the arch is smooth; and, malalignment of the fracture segment is not observed.

Good: deviation of the nasal bone is absent, overall shape of the arch is smooth; malalignment of the fracture segment is present, with either a one-segment irregularity or displacement.

Fair: deviation of the nasal bone is absent; overall shape of the arch is smooth; malalignment of the fracture segment is present; and, both bony irregularity and displacement is observed for each segment.

Poor: deviation of the nasal bone is present but at less than 20 degrees; overall shape of the arch is not smooth; and, bony irregularity and displacement is observed for each of two segments.

Very poor: deviation of the nasal bone is more than 20 de-

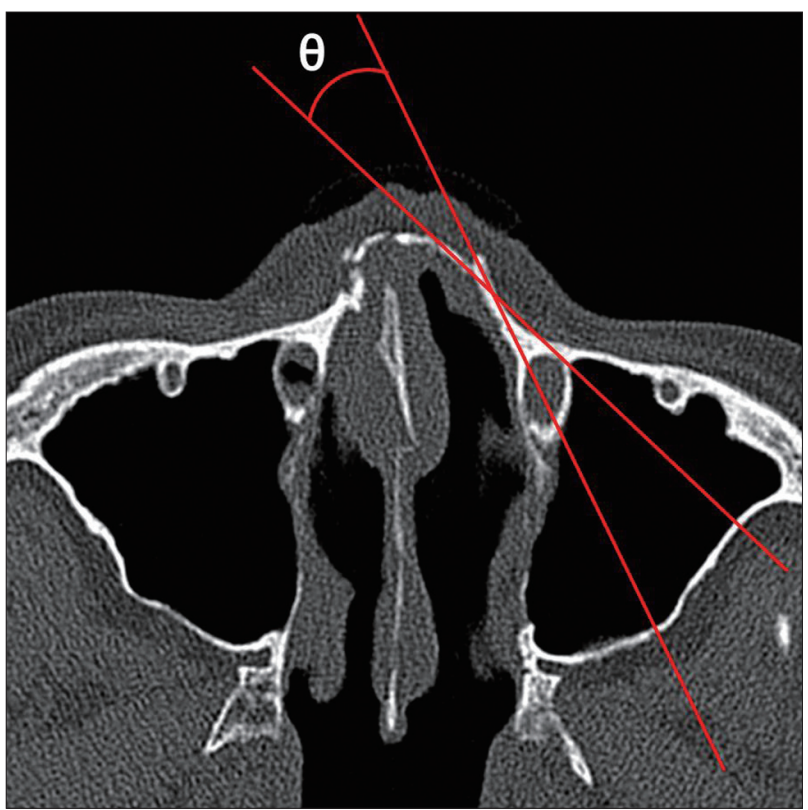

Fig. 1. Computed tomographic scan image of a nasal bone fracture. The angle $(\theta)$ between the nasal bone and the frontal process of the maxillary bone is used for deviation of nasal bone in postoperative evaluation.

Table 1. The classification criteria according to the result of closed reduction

\begin{tabular}{lcccc} 
& E & G & F & P \\
\hline Degree of deviation & - & - & - & Less than $20^{\circ}$ \\
Overall shape of arch & Smooth & Smooth & Smooth & Irregular \\
\hline Malalignment of fracture segment & - & + & + & Irregular \\
Bony irregularity & - & One segment or & One segment and & One or two segment and \\
Bony displacement & - & One segment & One segment & One or two segment \\
\hline
\end{tabular}

E, excellent; G, good; F, fair; P, poor; VP, very poor. 
grees, overall shape of the arch is not smooth; and, bony irregularity and displacement is observed for more than two segments (Table 1).

Nasal bone fracture classification by Stranc and Robertson [5] was used to characterize fracture type: frontal impact group type I (FI), frontal impact group type II (FII), lateral impact group type I (LI), lateral impact group type II (LII), and comminuted fracture group $(\mathrm{C})$.

We analyzed correlation between acute nasal bone remodeling and septal fracture or preexisting septal deviation by CT findings to estimate whether septal fracture or deviation could be improved by bone remodeling. Variables were compared using the chisquare test (SPSS ver. 19.0, IBM Co., Armonk, NY, USA). p-values less than 0.05 were considered to indicate statistical significance.

\section{RESULTS}

\section{Analysis of patients}

Of a total of 50 patients, 36 patients (72\%) were male and 14 patients $(28 \%)$ were female. Mean age was 32.1 years old (range, 7-82 years old). Per age groups, two patients (4\%) were under teen age, 12 patients (24\%) were teenagers, 19 patients (38\%) were in their twenties to thirties, 15 patients (30\%) were in their forties to fifties, and two patients (4\%) were in older than sixties.

\section{Types of fracture}

Eighteen patients (36\%) belonged to the frontal impact groups: type I (FI) ( $\mathrm{n}=14$ patients, $28 \%)$ and type II (FII) $(\mathrm{n}=4,8 \%)$. Twenty seven patients (54\%) belonged to the lateral impact groups: type I (LI) $(\mathrm{n}=13,26 \%)$ and type II (LII) $(\mathrm{n}=14,28 \%)$. The remaining 5 patients (10\%) were classified as being in the complex fracture (C) group.

\section{Results of reduction}

Immediately after the operation, CT-based outcomes in the FI group were fair for one patient (2\%), good for nine patients (18\%), and excellent for four patients (8\%). In the FII group, outcomes were fair for one patient (2\%) and good for three patients (6\%). In the LI group, outcomes were fair for two patient (4\%), good for eight patients (16\%), and excellent for three patients (6\%). In the LII group, outcomes were fair for two patients (4\%), good for nine patients (18\%), and excellent for three patients (6\%). In the $\mathrm{C}$ group, outcomes were poor for one patient (2\%), fair for two patients (4\%), and good for two patients (4\%) (Table 2).

Overall, the immediate postoperative CT outcomes were poor for one patient (2\%), fair for 8 patients (36\%), good for 31 patients (62\%), and excellent for 10 patients (20\%). At one month after operation, the outcomes were fair for one patient (2\%), good for 12patients (24\%), and excellent for 37 patients (74\%) (Table 3).

Comparing the change in reduction level, 20 patients (40\%) did not demonstrate any change in reduction level from the immediate postoperative period to 1 month after. Improved reduction level by bone reposition was observed in 30 patients (60\%; good-to-excellent for 22 patients, fair-to-excellent for five patient, fair-to-good for two patient, and poor-to-good for one patient) (Table 4).

According to fracture types, 7 out of 14 patients in the FI group showed improvements (fair-to-excellent for one patient and goodto-excellent for six patients). In the FII group, 3 out of 7 patients showed improvement (good-to-excellent for two patients and fair-

Table 2. Analysis of the results of reduction immediately after the operation according to fracture type

\begin{tabular}{lccccc} 
Fracture type & E & G & $F$ & $P$ & VP \\
Fl & 4 & 9 & 1 & 0 & 0 \\
FII & 0 & 3 & 1 & 0 & 0 \\
LI & 3 & 8 & 2 & 0 & 0 \\
LII & 3 & 9 & 2 & 0 & 0 \\
C & 0 & 2 & 2 & 1 & 0 \\
Total & 10 & 31 & 8 & 1 & 0 \\
\hline
\end{tabular}

E, excellent; G, good; F, fair; P, poor; VP, very poor; FI, frontal impact group type I; FII, frontal impact group type II; LI, lateral impact group type I; LII, lateral impact group type II; C, comminuted fracture group.

Table 3. Number of each group at day of surgery and one month after surgery

\begin{tabular}{lcc} 
Classification & Day of surgery & 1 month after surgery \\
Very poor & 0 & 0 \\
Poor & 1 & 0 \\
Fair & 8 & 1 \\
Good & 31 & 12 \\
Excellent & 10 & 37 \\
\hline
\end{tabular}


to-good for a single patient). In the LI group, 9 out of 13 patients (69\%) showed improvement (fair-to-excellent in two patients, and good-to-excellent in six patients). In the LII group, 9 out of 14 patients (64\%) showed improvement (fair-to-excellent in one patient, fair-to-good in one patient, and good-to-excellent in seven patients). In the comminuted fracture group, 3 out of 5 patients showed improvement (poor-to-good in one patient, fair-to-excellent in one patient, and good-to-excellent in one patient) (Table 5) (Fig. 2-5).

\section{Correlation between nasal bone remodeling and septal fracture or deviation}

There were no statistically significant relationships amongst nasal bone remodeling, preoperative septal fracture, or preexisting septal deviation (Table 6). Also, there were no statistically significant

Table 4. Change of result criteria at day of surgery and one month after surgery

\begin{tabular}{lc} 
Result of reduction & Number (\%) \\
Improvement cases & \\
$\mathrm{G} \rightarrow \mathrm{E}$ & $22(44)$ \\
$\mathrm{F} \rightarrow \mathrm{E}$ & $5(10)$ \\
$\mathrm{F} \rightarrow \mathrm{G}$ & $2(4)$ \\
$\mathrm{P} \rightarrow \mathrm{G}$ & $1(2)$ \\
Total number (\%) & $30(60)$ \\
No interval changed cases & \\
E $\rightarrow$ E & $10(20)$ \\
G $\rightarrow$ G & $9(18)$ \\
F $\rightarrow$ F & $1(2)$ \\
Total number (\%) & $20(40)$ \\
\hline
\end{tabular}

G, good; E, excellent; F, fair; P, poor.
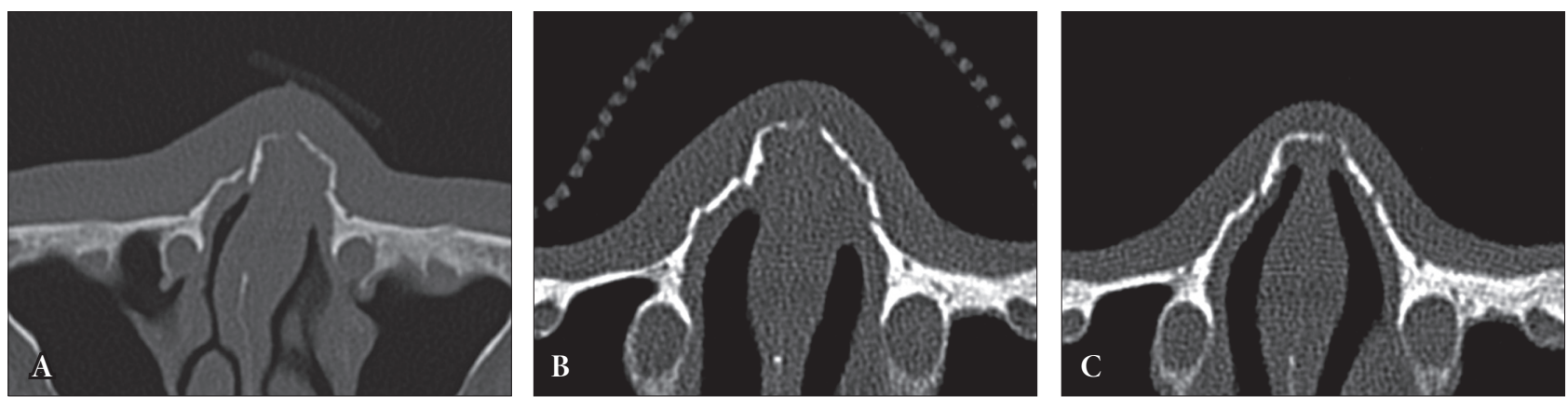

Fig. 2. Case 1. (A) Preoperative computed tomographic (CT) scan. (B) CT scan immediately after surgery. Evaluation is poor. (C) CT scan one month after surgery. Reevaluation is good. 

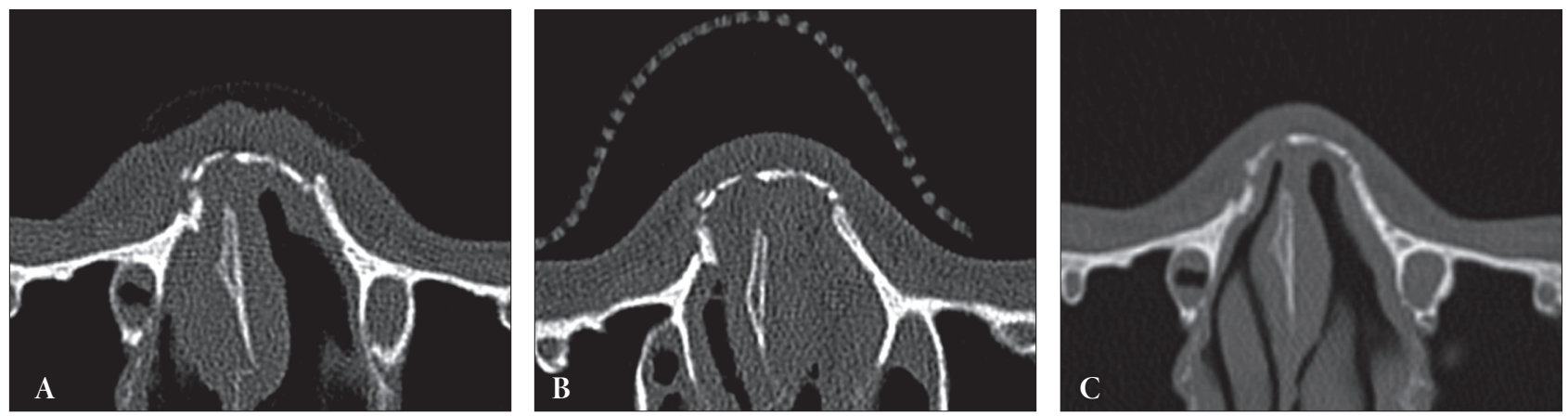

Fig. 3. Case 2. (A) Preoperative computed tomographic (CT) scan. (B) CT scan immediately after surgery. Evaluation is fair. (C) CT scan one month after surgery. Reevaluation is good.
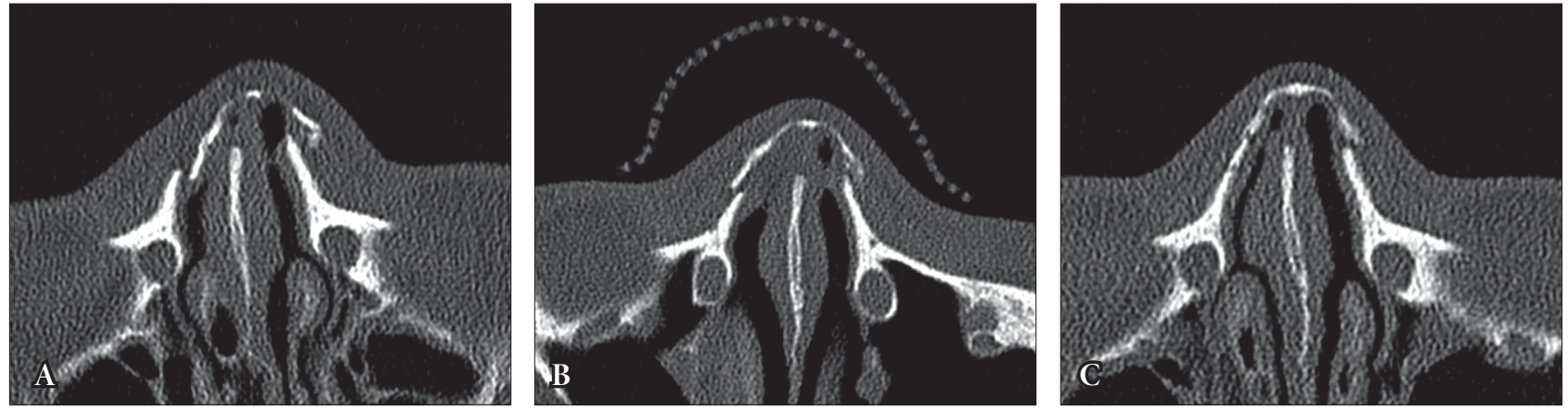

Fig. 4. Case 3. (A) Preoperative computed tomographic (CT) scan. (B) CT scan immediatelyafter surgery. Evaluation is fair. (C) CT scanone month after surgery. Reevaluation is excellent.
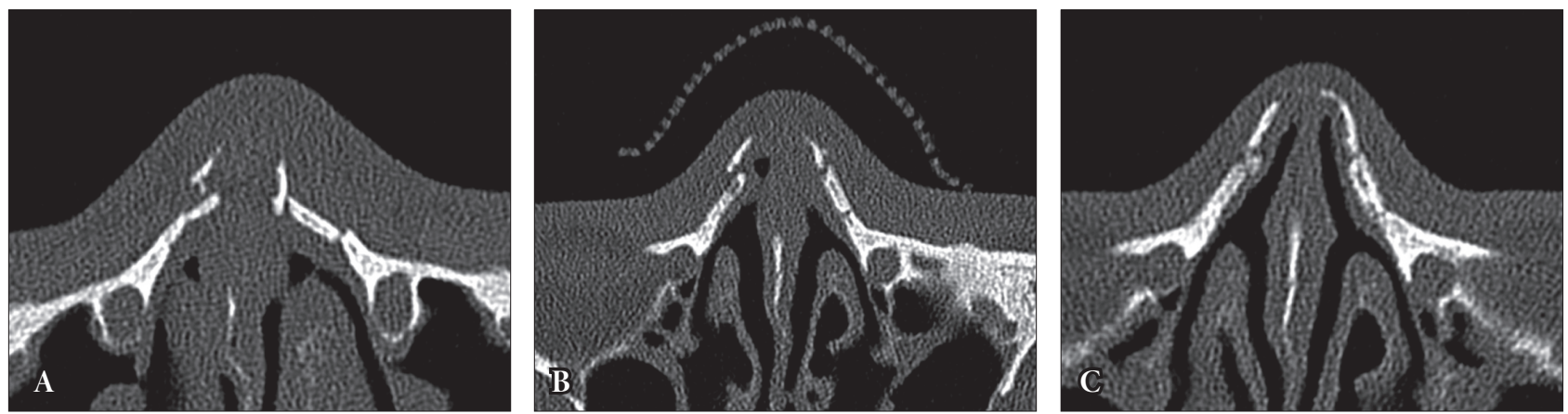

Fig. 5. Case 4. (A) Preoperative computed tomographic (CT) scan. (B) CT scan immediately after surgery. Evaluation is good. (C) CT scan one month after surgery. Reevaluation is excellent.

more accurate study of nasal bone fractures, determine the degree and direction of fracture, and the exact position of fracture. It facilitates reduction of the fracture and can also provide information regarding the damages to the surrounding area, including soft tissue and other facial bones [7].

Many studies have reported on the effectiveness of CT examination for diagnosis and postoperative evaluation of nasal bone fracture [8-10]. On the other hand, CT scanning is associated with higher radiation dose and its use is to be restrained as much as possible. Therefore, the repeat CT examinations were performed at the request of patients who expressed a desire to personally view the results of operation. Among younger patients who were still going facial bone growth, the parents worried about the possibility of abnormal nasal bone growth or nasal deformity after fracture reduction. In these situations, repeat CT scans offer several advantages including an increased confidence in postoperative 
Table 6. Results of nasal bone remodeling according to preoperative septal fracture or preexisting septal deviation

\begin{tabular}{lccc} 
Septal fracture or deviation & Cases & \multicolumn{2}{c}{ Nasal bone remodeling } \\
\cline { 3 - 4 } & & Improved cases & No interval changed cases \\
Preoperative & $39 / 50(78)$ & $24 / 39(62)$ & $15 / 39(38)$ \\
No preoperative & $11 / 50(22)$ & $6 / 11(55)$ & $5 / 11(45)$ \\
\hline
\end{tabular}

Values are presented as number (\%).

${ }^{a)}$ Chi-square test.

Table 7. Results of nasal bone remodeling according to remained septal deviation at one month after surgery

\begin{tabular}{|c|c|c|c|c|}
\hline \multirow{2}{*}{ Variable } & \multirow{2}{*}{ Cases } & \multicolumn{2}{|c|}{ At 1 month after operation } & \multirow{2}{*}{$p$-value ${ }^{a}$} \\
\hline & & Septal deviation & No septal deviation & \\
\hline Improved cases & $30(60)$ & 4/30 (13) & 26/30 (87) & 0.868 \\
\hline No interval changed cases & $20(40)$ & $3 / 20(15)$ & $17 / 20(85)$ & \\
\hline
\end{tabular}

Values are presented as number (\%).

${ }^{a)}$ Chi-square test.

outcomes for the patients and parents.

The outcomes was excellent for 10 patients (20\%), good for 31 patients (62\%), fair for 8 patients (16\%), and poor for a singles patient $(2 \%)$ on immediate postoperative CT studies. At one month after reduction, these outcomes had improved to excellent for 37 patients (74\%), good for 12 patients (24\%), and fair for one patient (2\%). Of all patients, 30 patients $(60 \%)$ showed immediate postoperative improvement of reduction. While 20 patients (40\%) showed no change, none of the closed reduction was associated with any worsening parameters.

Properly reduced nasal bone fragments are placed under selfsupporting force and are capable of maintaining the pyramidal configuration. Because nasal bone is membranous, the state of bone stump has an important effect on bone repair, more so when comparing reduction of long bones. The surrounding connective tissue and both layers of periosteum are important for progressive remodeling of reduced nasal bone. Therefore, both recovery of continuity by minimizing bony gap in the fracture segment and prevention of damage to the periosteum are important to obtaining good postoperative outcomes [11,12].

Patient (and parental) evaluation of postoperative outcomes can often be misleading because the external shape of the nose is under the influence of swelling and bone remodeling. Therefore, accurate diagnosis before the operation, precise reduction, careful management preventing of damage to periosteum and mucosa of the nasal bone, and thorough postoperative care are important in obtaining desired outcomes.

In this study, postoperative outcomes continued to improve during the first month after operation: in the FI group, seven patients of 14 patients; in the FII group, three patients of four patients; in the LI group, eight patients of 13 patients; in the LII group, four patients of 14 patients; in the C group, three patients of five patients. As septal fracture or preexisting septal deviation is often accompanied by nasal bone fracture, it is likely to develop complication such as postoperative nasal deformity with a negative effect on facial aesthetics [13]. We attempted to identify the relationship, if it exists, between nasal bone remodeling and the presence of septal deviation. However, no meaningful relationships were identified for nasal bone remodeling with either preoperative septal fracture or preexisting septal deviation. In addition, there were no statistically significant relationship between nasal bone remodeling and the presence of septal deviation at one month after operation.

In conclusion, it seems that reduced nasal segments tend to undergo progressive remodeling and that postoperative outcomes continued to improve over the first month. However, the current study has some limitations. The CT outcomes were not correlated with clinical examination parameters including the overall shape of external nose. Before the result of this study can be generalized, further studies are needed to overcome the small study size due to 
limited application of CT examination from concerns regarding ionizing radiation.

\section{REFERENCES}

1. Muraoka M, Nakai Y. Twenty years of statistics and observation of facial bone fracture. Acta Otolaryngol Suppl 1998;538:261-5.

2. Lee JH, Park WY, Nam HJ, Kim YH. Complications of the nasal bone fractures according to the stranc classification. J Korean Cleft PalateCraniofac Assoc 2008;9:62-6.

3. Han DG, Kim TS. The effect of half day nasal packing in results of closed reduction of nasal bone fracture. Arch Craniofac Surg 2012;13: $119-24$.

4. Motomura H, Muraoka M, Tetsuji MY, Ozawa MT, Nose MK. Changes in fresh nasal bone fractures with time on computed tomographic scans. Ann Plast Surg 2001;47:620-4.

5. Stranc MF, Robertson GA. A classification of injuries of the nasal skeleton. Ann Plast Surg 1979;2:468-74.
6. Mathes SJ, Hentz VR. Plastic surgery. 2nd ed. Philadelphia, PA: Saunders Elsevier; 2006.

7. Park CS, Suh CH, Seok EH, Chung WK, Byun US. Nasal bone fractures: evaluation with thin-section CP. J Korean Radiol Soc 1995;33: 197-203.

8. Manson PN, Markowitz B, Mirvis S, Dunham M, Yaremchuk M. Toward CT-based facial fracture treatment. Plast Reconstr Surg 1990;85: 202-12.

9. Pollock RA. Nasal trauma. Pathomechanics and surgical management of acute injuries. Clin Plast Surg 1992;19:133-47.

10. Kang SJ, Kim SK. The utility of thin section CT in pediatric nasal bone fracture. J Korean Cleft Palate-Craniofac Assoc 2003;4:104-6.

11. Neligan P. Plastic surgery. 3rd ed. Philadelphia, PA: Elsevier Saunders 2013.

12. Dahlin C, Linde A, Gottlow J, Nyman S. Healing of bone defects by guided tissue regeneration. Plast Reconstr Surg 1988;81:672-6.

13. Sam A, Deshmukh PT, Patil C, Jain S, Patil R. Nasal septal deviation and external nasal deformity: a correlative study of 100 cases. Indian J Otolaryngol Head Neck Surg 2012;64:312-8. 